\title{
The Aether of Spacetime Physics Is the Empty Set of Pure Mathematics
}

\section{Mohamed S. El Naschie}

Distinguished Professor, Department of Physics, Faculty of Science, University of Alexandria, Alexandria, Egypt

Correspondence to: Mohamed S. El Naschie, Chaossf@aol.com

Received: September 1, $2017 \quad$ Accepted: September 11, $2017 \quad$ Published: September 12, 2017

Copyright (c) 2017 by authors and Scientific Research Publishing Inc.

This work is licensed under the Creative Commons Attribution International License (CC BY 4.0).

http://creativecommons.org/licenses/by/4.0/

\section{c) (i) Open Access}

The present note is basically an announcement of a theoretical and experimental resolution of one of the most basic fundamental problems of physics, namely the very existence and reality of the Aether [1-11]. This is not only basic philosophy of science or insightful results in theoretical physics and cosmology [1-24] but more than that because it may lead to actually realizing the futuristic dream of free energy [25-28].

Our mathematical and indirect experimental verdict is that the Aether exists and it can be equated to the empty set of pure mathematics [3, 12-16]. More precisely the Aether may be understood for all mathematical and physical purposes as being identical to the empty set [8-14] underlying the Penrose fractal tessellation universe [17] which obeys the A. Connes' corresponding dimensional function of his noncommutative geometry [18-21].

It is then a relatively short distance to conclude from the above that cosmic dark energy is simply the energy stored in the Aether empty set [22-24] so that the agreement between the theoretical calculation and the numerous accurate cosmological measurements may be taken as an indirect experimental confirmation of this spectacular model for the Aether [10-16, 22-24]. In fact the mere fact that Penrose fractal tessellation is a well known and valid model for quasi crystals, i.e. a real material with five fold symmetry as well as for the cosmos at large $[10,17]$, is a sufficient fact to reinvigorate an old analogy between photons and phonon [7-11].

Seen in this larger imaginative new reality [7], we can go on confidently repeating what we have claimed for many years, that spacetime is physically real and may be regarded as a highly advanced material which can be harnessed to yield practically infinite clean, free energy via what we called a dark energy-Casimir nanotech reactor [15]. Such a reactor was thought of by many visionaries before but was never given anything like a firm mathematical and experimental justification as we did within the E-infinity theory proposal [21-28].

We leave it to the historians of science to relate our findings to that of the Tesla-Einstein discussion about the Aether [5]. However it is interesting to note that while Einstein started by thinking that his theory of relativity is a proof that the Aether could not exist or at a minimum is a totally unnecessary assumption, in his later years Einstein modified his stance and came much nearer to the thinking of N. Tesla who was a firm believer in the reality of the Aether [2, 5-7].

We think that although within the limited space of the present announcement we could not possibly give any detailed mathematical derivation, we should at least state some of the most important equations 
from which it became evident that $\mathrm{E}=\mathrm{mc}^{2}$ of Einstein implicitly included a recognition of the existence of the Aether by unconsciously including the energy of the Aether, i.e. a totally empty spacetime via the energy of the quantum wave as explained by the author on many previous occasions [3, 4, 9-18].

To come to this conclusion we may recall that we model the quantum pre-particle by the zero set $[3,6$, $9,11]$ and assign to it two dimensions, namely the topological dimension zero as befitting a point particle and the second Hausdorff dimension $\phi=(\sqrt{5}-1) / 2$ as obvious from Sir R. Penrose geometrical tessellation and Prof. A. Connes' dimensional function of the Penrose fractal universe [11, 17, 19, 20]. The pre-quantum wave on the other hand is the cobordism, i.e. the surface of the pre-particle [11-16]. Consequently it is an empty set and possesses two dimensions, similar to the zero set. The first is the Menger-Urysohn dimension minus one and the second is the Hausdorff dimension $\phi^{2}$ [11-20]. From the above we see that the cobordism of the pre-quantum wave must be given by a topological dimension minus two and a Hausdorff dimension $\phi^{3}[10,11]$. On the other hand the average Hausdorff dimension of spacetime is $4+\phi^{3}[10,11,21]$. Consequently the inverse of $4+\phi^{3}$ is $\phi^{3}$ [12-14]. That means on average the surface of the pre-quantum wave is spacetime itself $[12,13]$. That way we may see the quantum particle with its surrounding guiding pre-quantum wave as ripples in spacetime just as phonon are vibrational ripples in the quasi crystal [7-16]. Taking a bird's eye view of the entire situation, Aether, quantum field, quantum wave and spacetime become different names for very similar, in fact almost identical physico-mathematical entities [10-24].

It remains only to quantify the situation. Putting the five fold quasi crystal symmetry in a Kaluza-Klein five dimensional manifold we find a five dimensional zero set topological volume $\phi^{5}$ [16-21]. Thus the surface of $\phi^{5}$ is clearly an additive five dimensional area equal $5 \phi^{2}$ representing the pre-quantum wave $[3,4,10-16]$. Now we have shown in many previous publications [10-16] that $\phi^{5}$ is related to the ordinary measurable energy density of the universe and is found from the ordinary formula of classical kinetic energy when we let the velocity tend to that of light and find that $E(O)=(1 / 2)\left(m=\phi^{5}\right)\left(c^{2}\right)$ which amounts to about $4.5 \%$ of the energy density of special relativity, namely $\mathrm{E}=\mathrm{mc}^{2}$ divided by 22 $[10-16,22-24]$. As for the pre-quantum wave which is what gives us the dark energy sector and which cannot be measured directly because an empty set becomes non-empty and collapses on measurement [10-16, 22-24], one finds $E(D)=(1 / 2)(m=5)\left(c^{2}\right)$ which amounts to $95.5 \%$ of Einstein's density [12-24] that is approximately equal to Einstein's maximal density $\mathrm{mc}^{2}$ multiplied by 21 and divided again by 22 . The beauty of the present theory is summarized in unexpected confirmation of Einstein's famous formula, namely [22-24]

$$
\begin{aligned}
E & =E(O)+E(D) \\
& =\left[\left(\phi^{5} / 2\right) m c^{2}+\left(5 \phi^{2} / 2\right) m c^{2}\right] \\
& =(1 / 2)\left(\phi^{5}+5 \phi^{2}\right) m c^{2} \\
& =(1 / 2)(2) m c^{2} \\
& =E(\text { Einstein })
\end{aligned}
$$

Note as the most important that the above results are in complete agreement with the accurate cosmic measurements [11-16, 22-24]. In fact the present theory may be refined to account for dark matter energy and pure dark energy as well $[23,24]$.

From the preceding result we see that there is no doubt that the Aether exists and that it is basically a five dimensional empty set akin to the Penrose tessellation universe which is described by A. Connes' dimensional function of his noncommutative geometry as we indicated earlier on [17-19].

In a fourth coming book we will present a detailed analysis and discussion of the present announcement letter. 


\section{REFERENCES}

1. Sir. Penrose, R. (1991) The Mass of the Classical Vacuum. In: Saunders, S. and Browen, H., Ed., The Philosophy of the Vacuum, Clarendon Press, Oxford University, Oxford, 21-26.

2. Einstein, A. (1922) Ether and the Theory of Relativity. $5^{\text {th }}$ May 1920 at the University of Leiden, Holland. Methuen \& Co. Ltd., London. http://www-history.mcs.st-and.ac.uk/Extras/Einstein_ether.html

3. Marek-Crnjac, L. (2013) Cantorian Space-Time Theory: The Physics of Empty Sets in Connection with Quantum Entanglement and Dark Energy. Lambert Academic Publishing, Saarbrucken.

4. El Naschie, M.S. (2011) On the Philosophy of Being and Nothingness in Fundamental Physics. Nonlinear Science Letters $B, 1,4-5$.

5. Seifer, M.J. (2009) Tesla vs Einstein and the Birth of the New Physics. New Dawn, No. 113. http://www.newdawnmagazine.com/articles/tesla-vs-einstein-the-ether-the-birth-of-the-new-physics

6. Einstein, A. (2006) Einstein Relativity Theory Declares Aether Necessary (Youtube). https://www.youtube.com/watch?v=yH9vAIdMqng

7. Wliczek, F. and Krauss, L. (2017) Materiality of a Vacuum (YouTube). https://www.youtube.com/Watch?V=BBXDrNn6PVg

8. Auffray, J.-P. (2014) E-Infinity Dualities, Discontinuous Spacetimes, Xonic Quantum Physics and the Decisive Experiment. Journal of Modern Physics, 5, 1427-1436. https://doi.org/10.4236/jmp.2014.515144

9. Auffray, J.-P. (2015) E-Infinity, the Zero Set, Absolute Space and the Photon Spin. Journal of Modern Physics, 6, 536-545. https://doi.org/10.4236/jmp.2015.65058

10. Marek-Crnjac, L. (2015) On El Naschie's Fractal-Cantorian Space-Time and Dark Energy-A Tutorial Review. Natural Science, 7, 581-598. https://doi.org/10.4236/ns.2015.713058

11. El Naschie, M.S. (2016) The Self Similarity Equivalence Relation Connecting Newton's Energy with Einstein's Energy and Dark Energy. International Journal of Innovation in Science and Mathematics, 4, 42-57.

12. El Naschie, M.S. (2015) An Exact Mathematical Picture of Quantum Spacetime. Advances in Pure Mathematics, 5, 560-570. https://doi.org/10.4236/apm.2015.59052

13. El Naschie, M.S. (2016) The Emergence of Spacetime from the Quantum in Three Steps. Advances in Pure Mathematics, 6, 446-454. https://doi.org/10.4236/apm.2016.66032

14. El Naschie, M.S. (2016) On a Fractal Version of Witten's M-Theory. Journal of Astronomy \& Astrophysics, 6, 135-144.

15. El Naschie, M.S. (2015) On a Non-Perturbative Quantum Relativity Theory Leading to a Casimir-Dark Energy Nanotech Reactor Proposal. Open Journal of Applied Science, 5, 313-324.

https://doi.org/10.4236/ojapps.2015.57032s

16. El Naschie, M.S. (2017) Einstein-Kaluza Combined Spacetime as the Optimal and Simplest Framework to Compute and Understand Dark Matter, Pure Dark Energy and Measurable Ordinary Energy. Natural Science, 9, 241-244. https://doi.org/10.4236/ns.2017.98024

17. El Naschie, M.S. (1998) Penrose Universe and Cantorian Spacetime as a Model for Noncommutative Quantum Geometry. Chaos, Solitons \& Fractals, 9, 931-933.

18. El Naschie, M.S. (1998) Von Neumann Geometry and E-Infinity Quantum Spacetime. Chaos, Solitons \& Fractals, 9, 2023-2030.

19. Connes (1994) Noncommutative Geometry. Academic Press, San Diego.

20. El Naschie, M.S. (1994) On Certain "Empty" Cantor Sets and Their Dimensions. Chaos, Solitons \& Fractals, 4, 293-296. 
21. El Naschie, M.S. (2004) A Review of E-Infinity Theory and the Mass Spectrum of High Energy Particle Physics. Chaos, Solitons \& Fractals, 19, 209-236.

22. El Naschie, M.S. (2017) Kähler Dark Matter, Dark Energy, Cosmic Density and Their Coupling. Journal of Modern Physics, 7, 1953-1962.

23. El Naschie, M.S. (2017) A Combined Heterotic String and Kähler Manifold Elucidation of Ordinary Energy, Dark Matter, Olbers's Paradox and Pure Dark Energy Density of the Cosmos. Journal of Modern Physics, 8, 1101-1118. https://doi.org/10.4236/jmp.2017.87071

24. El Naschie, M.S. (2017) From a Dual Einstein-Kaluza Spacetime to 'tHooft Renormalon and the Reality of Accelerated Cosmic Expansion. Journal of Modern Physics, 8, 1319-1329. https://doi.org/10.4236/jmp.2017.88085

25. El Naschie, M.S. (2015) A Casimir-Dark Energy Nano Reactor Design-Phase I. Natural Science, 7, 287-298. https://doi.org/10.4236/ns.2015.76032

26. El Naschie, M.S. (2015) Casimir-Dark Energy Nano Reactor Design Proposal Based on Fractals. International Journal of Innovation Is Science and Mathematics, 3, 187-194.

27. El Naschie, M.S. (2015) A Cold Fusion-Casimir Energy Nano Reactor Proposal. World Journal of Nano Science and Engineering, 5, 49-56. https://doi.org/10.4236/wjnse.2015.52007

28. El Naschie, M.S. (2015) Kerr Black Hole Geometry Leading to Dark Matter and Dark Energy via E-Infinity Theory and the Possibility of Nano Spacetime Singularity Reactor. Natural Science, 7, 210-225. https://doi.org/10.4236/ns.2015.74024 (See also the literature referred to in this paper).

\section{Scientific Research Publishing}

Submit or recommend next manuscript to SCIRP and we will provide best service for you:

Accepting pre-submission inquiries through Email, Facebook, LinkedIn, Twitter, etc.

A wide selection of journals (inclusive of 9 subjects, more than 200 journals)

Providing 24-hour high-quality service

User-friendly online submission system

Fair and swift peer-review system

Efficient typesetting and proofreading procedure

Display of the result of downloads and visits, as well as the number of cited articles

Maximum dissemination of your research work

Submit your manuscript at: http://papersubmission.scirp.org/

Or contact ns@scirp.org 